\title{
Computer Assisted Dental Implantology: A New Method and a Clinical Validation
}

\author{
Julien Dutreuil $^{1}$, François Goulette ${ }^{1}$ and Claude Laurgeau ${ }^{1}$ \\ Jaime Clavero Zoreda ${ }^{2}$ and Stefan Lundgren ${ }^{2}$ \\ ${ }^{1}$ Centre de Robotique, Ecole des Mines de Paris, 60 boulevard Saint Michel, \\ 75272 Paris Cedex 06, France \\ \{dutreuil, goulette, laurgeau\}@caor.esnmp.fr \\ ${ }^{2}$ Department of Oral and Maxillofacial Surgery, Umeå Universitet, 90187 Umeå, Sweden \\ jclavero.z@tbc.es, stefan.lundgren@odont.umu.se
}

\begin{abstract}
This paper presents a new method for dental implant surgery. A preoperative planning software is used to work with CT scanner data. Implant fixtures are placed with the help of a 3D reconstructed model of the patient's jaw. An accurate robot is then used to drill a jaw splint, at the locations determined with the planning software, in order to make a surgical guide. A validation case of this new technique is also presented.
\end{abstract}

\section{Introduction}

Dental implants are used in maxillofacial restoration to replace a tooth or a set of teeth. One implant is composed of three parts : a titanium fixture surgically placed into the patient's jaw, a prosthetic crown replacing the missing tooth, and a mechanical part connected between the fixture and the crown, the abutment.

The success of a dental implantation depends directly on the localisation of the fixture in the patient's jaw bone. Firstly, the good adequacy between the axis of the fixture (surgical axis) and the axis of the prosthetic tooth (prosthetic axis) is critical for the good setting of the implant : the angle between both axes must be as small as possible in order to minimise lateral constraints transmitted from the crown to the abutment and then to the fixture [1]. Secondly, the fixture must be precisely implanted in hard cortical bone to assure a good stability. The surgery also requires great accuracy because of particularly sensitive anatomic structures in the neighbourhood of these implants (the mandibular nerve and the maxillary sinus) [1,2].

The new technique we propose improves the accuracy in the fixture placement. It uses a pre-operative planning phase for virtual placement of the fixture, and an accurate robot to drill a jaw splint, exact negative shape of the patient's jaw, in order to create a surgical guide.

Other solutions have already been proposed to improve the accuracy of the fixture placement: using a robot for drilling a splint $[1,3,4]$, using stereo-lithography to build the splint from CT scans $[5,6]$, or using a navigation system during the surgery, coupled with scanner data $[7,8]$. 


\section{Overview of the New Method}

This method consists in a 4-stage procedure, presented below and on figure 1 .

Stage $1:$ a denture impression of the patient's jaw is taken. It is used to make a plaster model of the jaw, and a splint on which the missing teeth are replaced by crowns. Radio-opaque balls are placed at precise locations on the splint, to provide scanner markers [9].

Stage 2 : a CT scan of the patient is taken, with the splint placed in the mouth. The use of CT scans is very classical in dental implantology, because they can provide complete $3 \mathrm{D}$ data by tomographic reconstruction, with an excellent sensitivity to bone tissues density $[9,10]$.

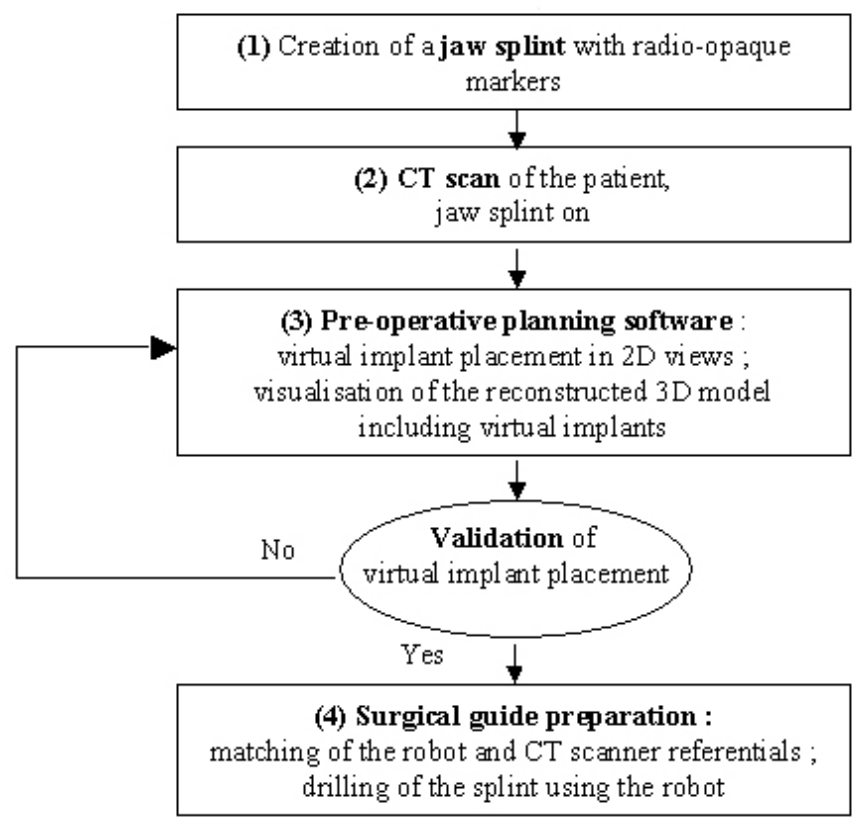

Fig. 1. Sketch of the new method

Stage 3 : the clinician uses the pre-operative planning software and the CT scan data to precisely choose the location of virtual implants. Visual validation of the locations planned is made with the help of $3 \mathrm{D}$ reconstructed views of the patient's jaw.

Stage 4 : the splint is placed on the plaster model which is fixed on the robot work plan. Radio-opaque balls located on the splint are visible directly, on the splint and on the CT scanner data : the matching between the robot referential and the CT data referential may be performed. The result of the matching is combined with the information of the desired implant location, expressed in the scanner referential, to compute the implant location in the robot referential. Finally, the splint is drilled by the robot at this precise location to make the surgical guide. 


\section{Detailed Technical Description of the Method}

Some of the stages described above need additional description : the pre-operative planning stage, the method used for 3D visualisation of the scanner data, and the making of the surgical guide including the drilling of the splint.

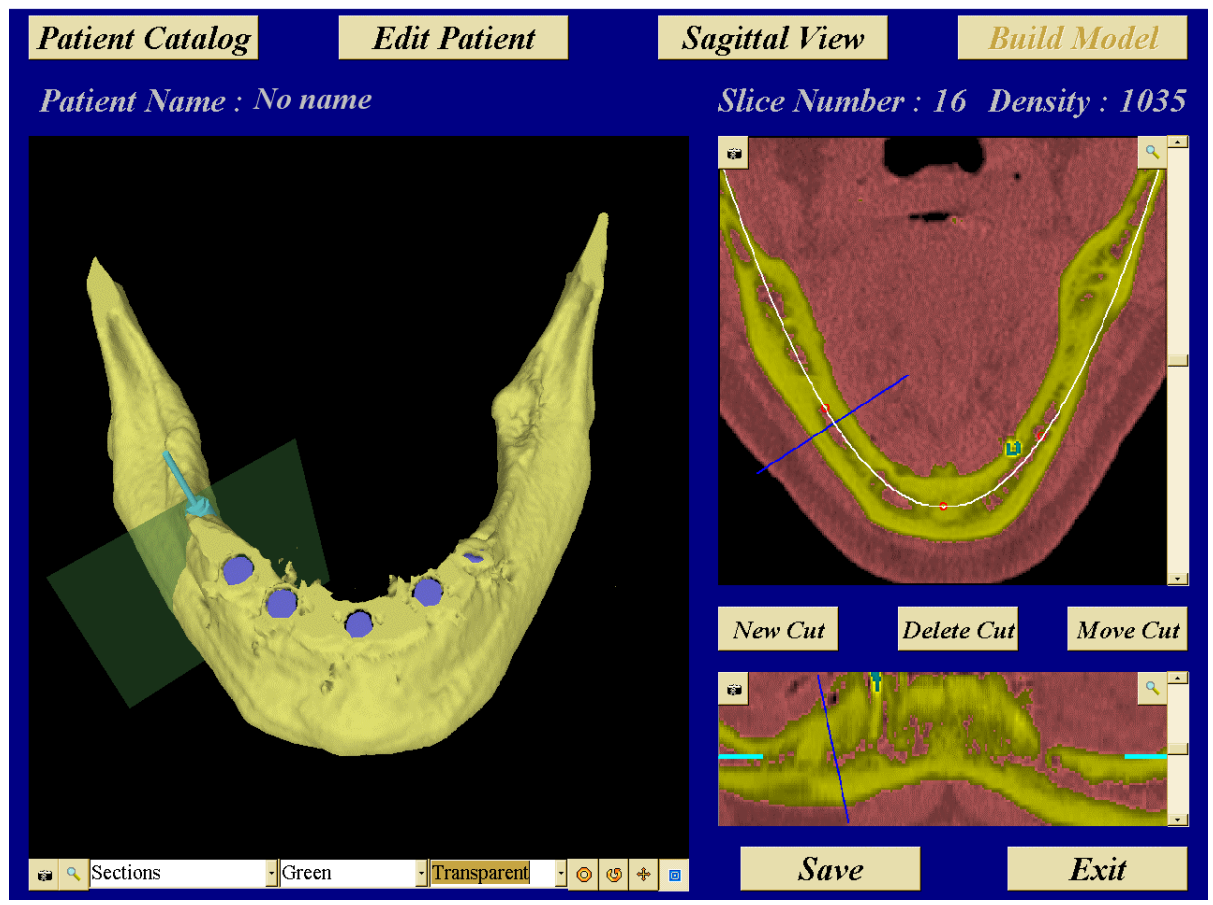

Fig. 2. General view from the pre-operative planning software

(left : 3D view reconstructed from 2D scanner images; top-right : occlusal view ; bottom-right : panoramic view)

\subsection{Pre-operative Planning}

To prepare the surgery, a dedicated software that has been especially developed to be adapted to the needs of dental implant surgeons is used (Figure 2). The planning stage consists in identifying the best location to place implants inside the patient's jaw, according to the ideal prosthesis, in order to improve the medical result [11]. The software provides tools and cut views interpolated from the data to optimise this location process.

In a very classical way, dental surgeons work in a local referential fixed on the patient's jaw. This referential defines 3 classical views : the occlusal view, the panoramic view, and the vestibo lingual view.

The occlusal plane is the key reference in the patient's mouth. It is defined by the plane of teeth occlusion. Scanner data are usually acquired in planes parallel to 
this occlusal plane, so we assimilate occlusal views with these scanner pictures (Figure 2, top-right).

The panoramic view is defined as a view perpendicular to the occlusal plane, curved on a specific way following the jaw shape of each patient. In the software (figure 2, bottom-right), it is defined in the occlusal plane with the help of a 2D curve, which is a $3^{\text {rd }}$ order polynomial. This polynomial is generated by three control points, with a null derivative constraint on the middle point.

The vestibo lingual view is defined locally, as a planar cut view positioned along the panoramic curve, locally perpendicular to this curve. Initially perpendicular to the occlusal plane, it can be inclined from the direction perpendicular to the occlusal plane with a few degrees $\left(+/-15^{\circ}\right)$, in order to adapt the view to the specific anatomy of the patient and to get more information.

On the planning software, the surgeon visualises the occlusal views, defines the panoramic view by moving the control points, and chooses the vestibo lingual cut views. Implant placements are usually performed initially in the vestibo lingual views. The position, orientation, length and diameter of the implant may be defined interactively, in each view, with a visual feedback in all the graphical windows.

\subsection{D Visualisation of Scanner Data}

The 3D model of the patient anatomy gives helpful information on implants location and inclination. In order to build such a $3 \mathrm{D}$ model, a pre-processing segmentation stage is performed to identify, for each pixel, the kind of human tissue to which it belongs. For dental surgery, there are soft tissues (mucosa, skin, muscles, fat), bone tissues (soft bone, hard cortical bone, teeth) and metal objects (surgical screws, implants, radio-opaque markers...).

The good sensitivity of CT scans for bone tissues makes it possible to use a simple threshold to segment the raw images, after a simple filtering operation (low pass filter), to eliminate the background noise in the measured data. The comparison of scanner images pixel intensities to standard values gives a good evaluation of the pixel nature. Data obtained by CT scans are expressed in Hounsfield Units (HU), normalised with the air density (-1000 HU) and the water density $(0 \mathrm{HU})$. Therefore, the threshold values are identified universal constants: e.g., bone generally ranged from $200 \mathrm{HU}$ to $1500 \mathrm{HU}$.

The measure points are regularly distributed on a 3D grid. After the segmentation operation, we generate a skin surface model by extracting a local mesh on each voxel of this 3D grid. Locally, the skin triangulation mesh is obtained using a method based on the "marching-cube" algorithm [12].

For each voxel, the mesh configuration is identified by looking at the tissues recognised on each vertex. For all segments including an intersection with the tissue, the intersection location is estimated by linear interpolation of the threshold between the 2 extremity values. Instead of a reduced number of cases identified by symmetry analysis, we use a complete reference table (256 cases), which speeds up the algorithm and minimises the risk of generating unclosed meshes.

Once the model is built, a post-processing stage is necessary. Generated models are very large. For example, a poor resolution scanner exam ( 256 x 256 pixels per picture with 30 pictures) can generate more than 250,000 triangles just for bone 
tissues. For high resolution ones (512 x 512 pixels per picture with 50 pictures), the number of generated triangles can be over 1,500,000. In order to work with such models on a simple PC, a simplification stage is necessary.

Different solutions are possible for mesh simplification, as segment or vertex decimation methods [13] or use of a decimation criterion. In our software, a basic method using vertex decimation, with a threshold criterion on a propagated angular deformation of vertex normals has been implemented. For the next version of the software, a segment decimation method using a parametric representation of the local surface is considered [14].

\subsection{Drilling of the Surgical Guide}

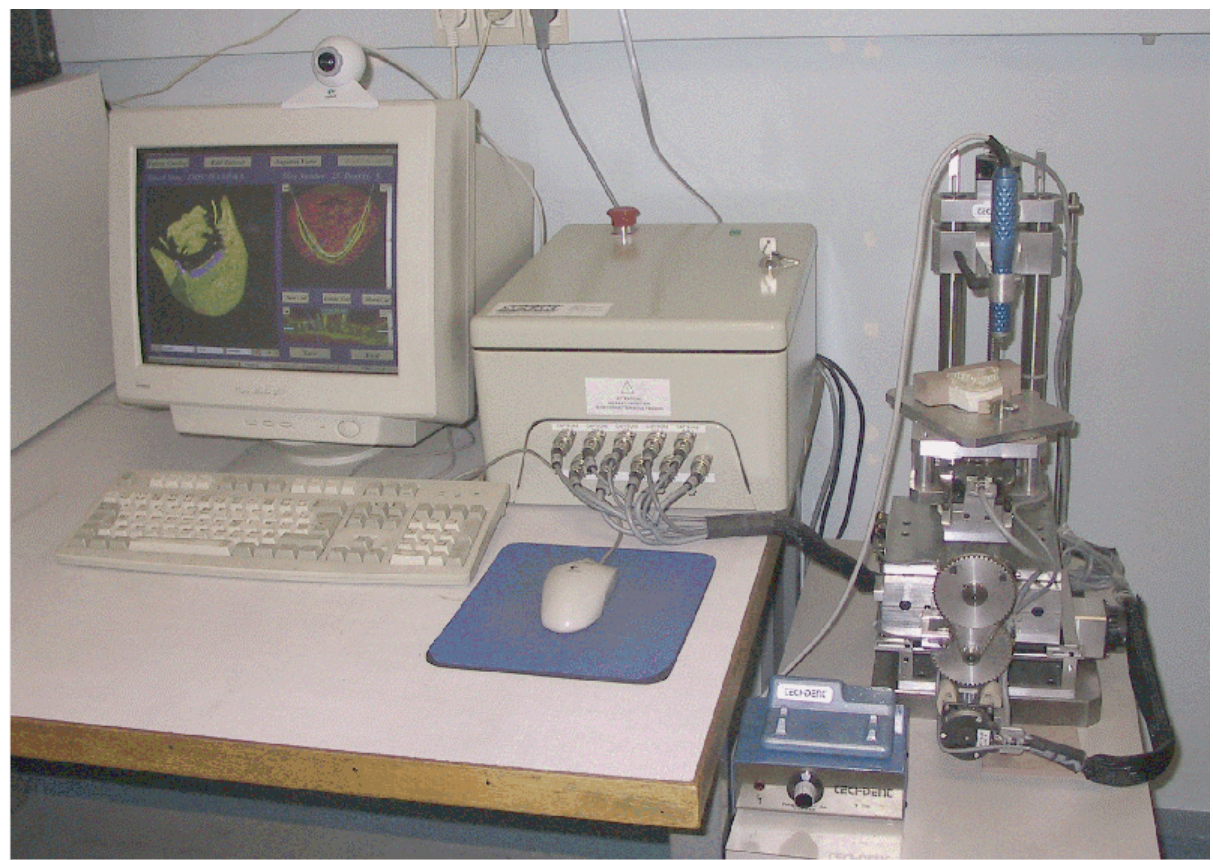

Fig. 3. Complete processing workbench, from pre-operative planning to splint drilling

As presented above, the jaw splint is placed in the mouth of the patient during the scanner acquisition. Radio-opaque balls are placed on this splint, at several specific positions regularly spaced along the jaw arch. These radio-opaque balls are visible in both the scanner images and the jaw splint.

After the virtual implant placement is done, the plaster model is attached to the robot work plan, and the splint is adjusted on the plaster model. The robot is an accurate numerical command machine, with five degrees of freedom ( 3 translations and 2 rotations). It has a $0.04 \mathrm{~mm}$ translation accuracy, and a $0.15^{\circ}$ rotation accuracy (Figure 3). 
By pointing the radio-opaque balls with the robot, the location of these balls in the robot referential is determined with an accuracy close to $0.3 \mathrm{~mm}$; by pointing them in the CT scanner images, their location in the scanner referential is also determined, with an accuracy close to $0.25 \mathrm{~mm}$ (only limited by the scanner definition).

The radio-opaque balls locations in the two referentials (robot referential and scanner referential) define two sets of control points that have to be matched, by identification of 3 rotation and 3 translation parameters defining the referential frame transformation. This is a non linear problem, which is solved using a least squares minimisation of the sum of distances between each couple of points. The classical Levenberg Marquardt algorithm [15] is used, instead of a simple gradient method to prevent low convergence trouble caused by flat potentials.

We can evaluate that the global accuracy of the complete treatment, from the planning phase to the surgery as presented on figure 3 , is lower than $1 \mathrm{~mm}$ in location and $1^{\circ}$ in orientation.

\section{Clinical Validation}

A first case has been treated with this new protocol, at the department of oral and maxillo-facial surgery of Umeå University, in Sweden. The patient was an upper jaw edentulous woman. Because of a strong bone resorption, she had a bone graft before the implant surgery.

It is noteworthy that, even if the patient was treated with our new experimental method, there was no additional risk compared to the classical method, as has been pointed out elsewhere for a similar method [1]: a visual expertise was performed by the surgeon on the surgical guide before the surgery.

A 8-implant treatment was planned, using the pre-operative planning software, visible on figure 4-a ; figure 4-b shows the robot drilling the splint; on figure 4-c, one can see the patient during the surgery, with the surgical guide placed in the mouth, right before drilling the jaw ; figure 4-d displays the position of the fixture implants placed in the jaw after drilling.

This first operation was a success, validating our method : all implants were precisely placed at the right location.

\section{Conclusion}

A new method for dental implantology has been presented, which is based on the use of a surgical guide, CT scanner imaging, a pre-operative planning software, a robot to drill the splint and a method based on radio-opaque markers to match the implant virtual positions and the actual drilling directions in the splint.

The first clinical case demonstrated the simplicity and the efficiency of the method. Further work is planned with a clinical validation campaign. 


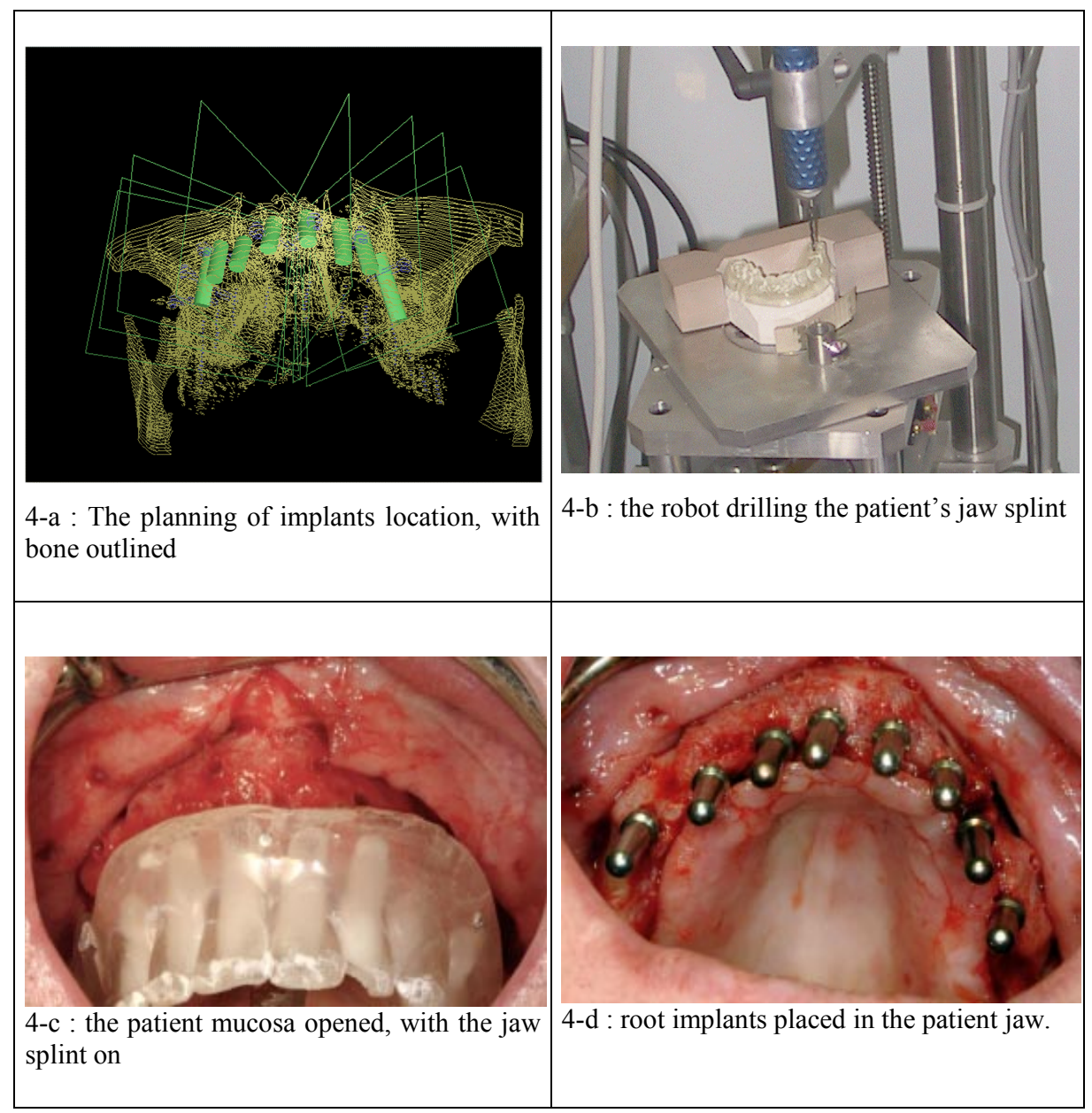

Fig. 4. The first validation case.

\section{Acknowledgments}

This work has been partly supported by the VISIMPLANT Leonardo da Vinci European program (partners: Ecole des Mines de Paris, Umeå University, Techdent, SFO, Imperial College). 


\section{References}

1. T. Fortin, J.P. Coudert, G. Champleboux et al. : "Computer-assisted dental implant surgery using computed tomography", Journal of image guided surgery, no. 1, p. 53-58, 1995

2. S.L. Bass : "The effects of preoperative resorption and jaw anatomy on implant success: a report of 303 cases". Clin. Oral Impl. Res., no. 2, p. 193-198.

3. G. Champleboux, E. Blanchet, T. Fortin et al. : "A fast, accurate and easy method to position oral implants using computed tomography". In Proc. Computer Assisted Radiology CAR'98, Elsevier Science, H.U.Lemke Ed., 1998.

4. K. Verstreken, J. van Cleynenbreugel, G. Marchal et al. : "An image guided planning system for oral implant surgery". Proc. of Computer Aided Radiology - CAR '96, p. 888-893, 1996.

5. V. Hietschold, W. Harzer, L. Eckhardt et al. : "Stereolithography of the occlusion plane using MR-tomographic imaging of the set of teeth". In Proc. Computer Assisted Radiology, 1996.

6. J. Lambrecht, C. Besimo, W. Müller et al. : "Precision of presurgical implantological planning with digitised CT and Scanora". In Proc. Computer Assisted Radiology, 1996.

7. P. Solar, S. Rodinger, C. Ulm et al. : "A computer-aided navigation system for oral implants using 3D-CT reconstructions and real time video projection", Int. Conf on Computer Assisted Radiology, 1996.

8. W. Birkfellner, F. Wanschitz, F. Watzinger et al. : "Accuracy of a Navigation System for Computer-Aided Oral Implantology", Proc. MICCAI'00, Lecture Notes in Computer Sciences no. 1935, Springer Verlag, p. 1061-1067, 2000.

9. N. L. Frederiksen : "Diagnostic imaging in dental implantology", Oral surgery, oral medicine, oral pathology, vol. 80, no. 5, p. 540-554, 1995.

10. N. Bellaiche, D. Doyon : "La tomodensimétrie dans le bilan pré-opératoire en implantologie orale", Journal de la Radiologie, tome 73, n¹, 1992.

11. K. Verstreken, J. Van Cleynenbreugel, K. Mertens et al. : "An Image-Guided Planning System for Endosseous Oral Implants", IEEE Transactions on Medical Imaging, 17(5): p. 842-852, 1998

12. W.E. Lorensen, H.E. Cline : "Marching Cube: A High Resolution 3-D Surface Construction Algorithm", Computer Graphics, 21(3): p. 163-169, 1987

13. W.J. Schroeder, J.A. Zarge, W.E. Lorensen : "Decimation of Triangle Meshes", Computer Graphics, 26 (2), p 55-64, 1992.

14. M.J. Diaz, F. Hecht, “Anisotropic Surface Mesh Generation”, INRIA, MENUSIN project, research report $n^{\circ} 2672,1995$.

15. D.W. Marquardt, Journal of the society for industrial and applied mathematics, vol. 11, p. 431-441, 1963. 